\title{
Experimental study of energy delivered to the filaments in high pressure nanosecond surface discharge
}

\author{
Chenyang Ding*, Antonin Jean ${ }^{\dagger}$, Sergey Shcherbanev ${ }^{\ddagger}$ \\ Laboratory of Plasma Physics (CNRS, Ecole Polytechnique, Univ. Paris-Sud, Sorbonne Université, l'Institut \\ Polytechnque de Paris), Ecole Polytechnique, route de Saclay, 91128 Palaiseau, France \\ Igor Selivonin $^{\S}$, Ivan Moralev ${ }^{\text {II }}$ \\ Joint Institute for High Temperatures of the Russian Academy of Sciences, Moscow, 125412, Russia \\ Nicolay Popov"l \\ Skobeltsyn Institute of Nuclear Physics, Moscow State University, Moscow, 119991, Russia. Joint Institute for High \\ Temperatures of the Russian Academy of Sciences, Moscow, 125412, Russia \\ Svetlana Starikovskaia** \\ Laboratory of Plasma Physics (CNRS, Ecole Polytechnique, Univ. Paris-Sud, Sorbonne Université, l'Institut \\ Polytechnque de Paris), Ecole Polytechnique, route de Saclay, 91128 Palaiseau, France
}

\section{Introduction}

Nanosecond surface dielectric barrier discharge (nSDBD) was suggested for ignition of combustible mixtures in 11,2]. During the experiments at high pressures [3-5], it was found that a severe transformation of a single-shot nanosecond surface streamer discharge is observed at increasing gas pressure and/or voltage. A regular structure of tens of contracted channels - filaments appeared from the edge of the high-voltage electrode and propagated in the direction perpendicular to the edge of the high-voltage electrode, the number of filaments being $4-5$ times less than the number of streamers. The effect was first observed for negative polarity discharge [3] and later it was found that the streamer-to-filament transition is a common feature for all observed molecular gases and mixtures containing molecular gases [5]. Extremely high electron density, $n_{e}=10^{18}-10^{19} \mathrm{~cm}^{-3}$ and slow electron density decay, tens of nanoseconds, were reported for $\mathrm{N}_{2}: \mathrm{H}_{2}$ mixture [4]. During last $3-5$ years, a few groups [6-8] observed similar high densities of electrons with a slow decay rate in atmospheric pressure plasma pin-to-pin discharges driven by repetitive high frequency (typically at least tens of $\mathrm{kHz}$ ) nanosecond pulses; constriction of the discharges corresponding to sharp increase of the electron density in a nanosecond time scale has been experimentally observed. Filamentary surface dielectric barrier discharge provides similar plasma properties in the filaments but instead of a single shot channel, multiply equidistant filaments (30-50 in our case) appear along the edge of the high-voltage electrode. A regular "grid" of the filaments results in efficient and stable ignition of combustible mixtures. Our recent experiments shown high, more than $7 \mathrm{eV} /$ particle, specific delivered energy for the filaments in air initiated by pulses of negative polarity 30-45 $\mathrm{kV}$ on the electrode at gas pressure equal to 8 bar. Developed physical model of the filamentary nanosecond surface DBD suggests extra-raid fast gas heating (tens of kK during parts of nanoseconds) in discharges at high electric field and high energy density at elevated pressures. In this case the efficiency of ignition is explained by high temperature and quasi-equilibrium plasma formed in the filaments; high density of $\mathrm{O}$-atoms and high hydrodynamic perturbations in the vicinity of each filaments. One of open questions is the initial stage, namely how streamer-to-filament transition happens and what is a physical reason of sub-nanosecond single-shot construction. The aim of the present work is an experimental study of appearance of the filaments using microimaging; of the value of specific delivered energy and study of character of hydrodynamic perturbations for the filamentary nSDBD discharge of positive polarity.

\footnotetext{
*Graduate student, Laboratory of Plasma Physics, CNRS, Ecole Polytechnique.

†Undergraduate student, Laboratory of Plasma Physics, CNRS, Ecole Polytechnique.

${ }^{\ddagger}$ Postdoctoral researcher, CNRS, Ecole Polytechnique, current address: CAPS Lab, Dpt of Mechanical and Process Engineering, ETH Zurich, Switzerland.

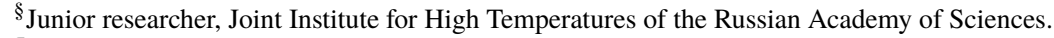

"Leading researcher, Joint Institute for High Temperatures of the Russian Academy of Sciences.

"Leading researcher, Skobeltsyn Institute of Nuclear Physics, Moscow State University.

** Leading researcher, CNRS, Ecole Polytechnique.
} 


\section{Experimental Setup}

A. Nanosecond surface dielectric barrier discharge

Figure 1 presents the schematic of the electrode system. A gear-shaped metal disk, $20 \mathrm{~mm}$ in diameter, served as the high-voltage (HV) electrode in the experiments. The low-voltage (LV) ground electrode has $20 \mathrm{~mm}$ internal diameter and $46 \mathrm{~mm}$ external diameter. A thin PVC film ( $\varepsilon=3-3.5, d=0.3 \mathrm{~mm}$ in thickness), serving as the dielectric, was glued to the ground electrode by Geocel FIXER Mate silicon glue which has the similar permittivity.
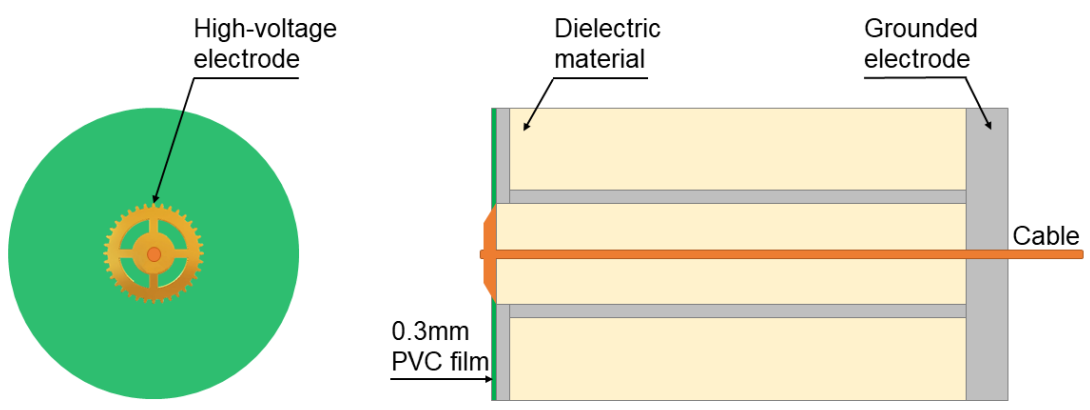

Fig. 1 Schematic of the electrode system.

The electrode system was installed in a constant volume high-pressure chamber which allows working in a wide pressure range from 100 mbar to 12 bar. The discharge chamber had 3 quartz windows, allowing the optical measurement experiments. The electrode was connected to the FPG20-03PM generator (FID technology) by a $30 \mathrm{~m}$ long $50 \Omega$ coaxial cable. The high-voltage pulse with $20 \mathrm{~ns}$ in duration, $2 \mathrm{~ns}$ rise time, positive polarity was delivered through the cable to the HV electrode. The oscilloscope Lecroy was used to record the voltage waveforms. The integrated ICCD images (200-800 nm sensitivity range) were taken by the Pi-Max4 ICCD camera (Princeton Instruments). For macroimaging, the ICCD was connected to a long distance microscope (LaVision QM1, Maksutov-Cassegrain catadioptric) equipped with a Barlow lens. The best achieved resolution was $1.1 \mu \mathrm{m} / \mathrm{px}$. For imaging, the resolution between 3.6 and $7.6 \mu \mathrm{m} / \mathrm{px}$ was used. The experiments were performed in a single shot regime. Before each experiment, the discharge cell was pumped down to $10^{-2}$ Torr. $\mathrm{N}_{2}$ and $\mathrm{O}_{2}$ (Air Liquid) with less than $100 \mathrm{ppm}$ of impurities were used to prepare the mixtures.

\section{B. Particle image velocimetry (PIV) measurement}

To study the hydrodynamic and thermal effects of filamentary nanosecond surface dielectric barrier discharge, particle image velocimetry (PIV) experiments were conducted. LaVision Flow Master PIV system wasused for the measurements. The experiments were performed in the configurations shown in Figure2

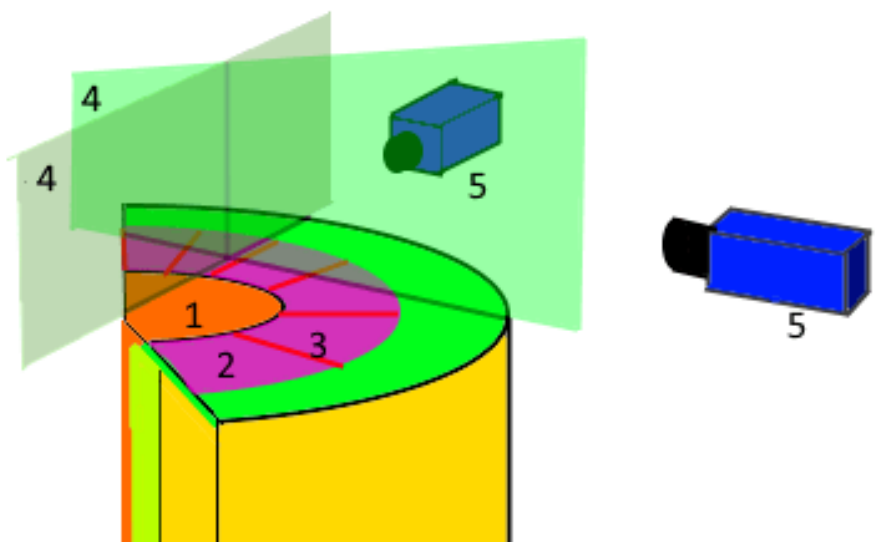

Fig. 2 Schematic of the PIV experiment. 
Laser frequency was equal to the discharge repetitive frequency, $7 \mathrm{~Hz}$. Laser sheet was aligned in one of two planes normal to the dielectric surface. The particle images were captured by a CCD camera (4Mpx, 16 bit) with the lens central line being normal to the laser sheet. The laser pulse was synchronized with discharge with a certain delay, the typical delay between the laser pulses (inter-frame delay) was varied from 0.2 to $100 \mu$ s depending of the stage of the flow evolution. The mean velocity distribution was obtained as a result of averaging 70 instanteneous vector fields from the individual measurements. Three-dimensional vector fields were acquired by shifting the discharge cell in the direction normal to the laser sheet and by combining the data from the two views. Cross-correlation was calculated in $32 \times 32$ px windows with 50\% overlap, consequent signal-to noise and smoothing filters were applied.

\section{Results and Discussion}

Microscopic images of streamer-to-filament transition, experimental study of the specific delivered energy and experimental study of the hydrodynamic and thermal effects of filamentary nanosecond surface dielectric barrier discharge are presented below.

\section{A. Microscopic images of filamentary discharge}

To study the developemnt of a streamer-to-filament transition and to observe the changes of the general structure of the discharge with voltage, two different configurations of the high-voltage electrode were used. For time-resolved measurements, a gear-like high-voltage electrode $20 \mathrm{~mm}$ in diameter similar to the electrode used in [4] was used. For time-integrated images, a smooth edge electrode of the same diameter was used. All the experiments on microimaging were performed in nitrogen at $P=6$ bar pressure; positive and negative polarities of the high-voltage pulse were compared.

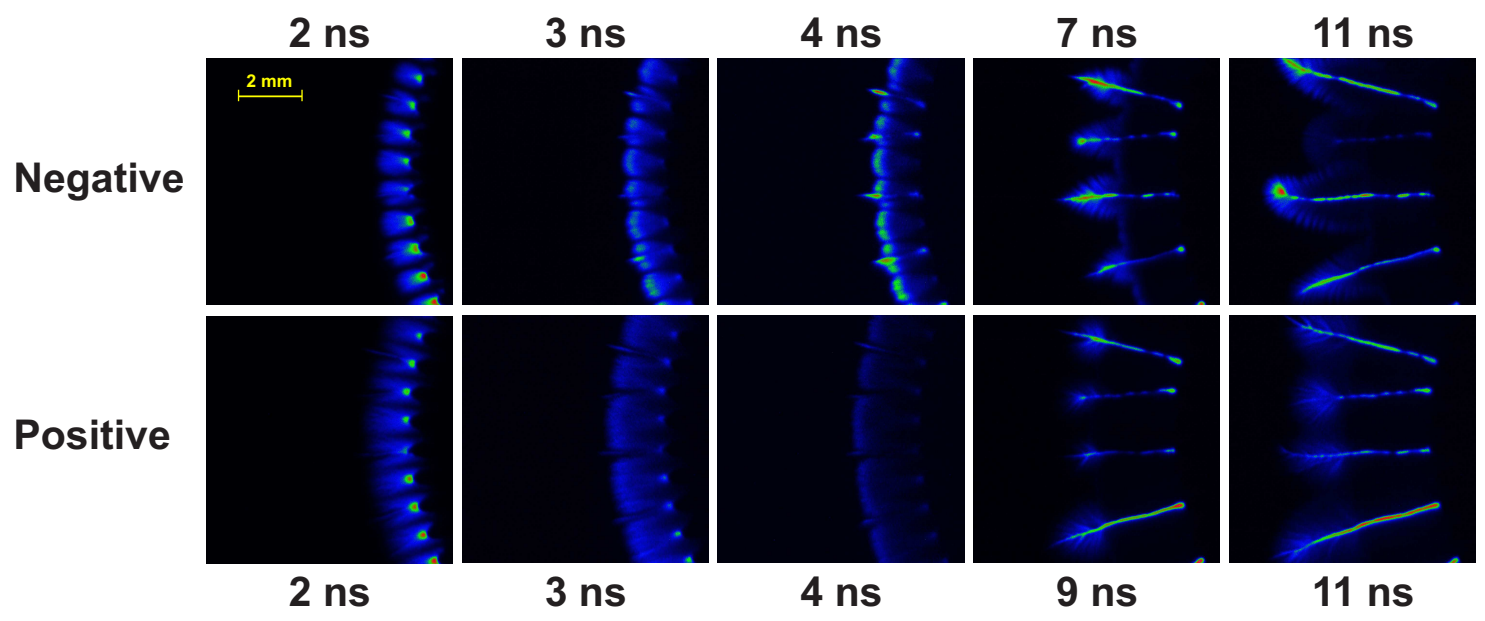

Fig. 3 ICCD images of streamer-to-filament transition $(P=6 \mathrm{bar}, U= \pm 25 \mathrm{kV})$ in nitrogen. Camera gate is $1 \mathrm{~ns}$.

Dynamics of the discharge development is given by Figure 3 . The voltage amplitude was fixed and equal to $\pm 25 \mathrm{kV}$. The camera gate was equal to $1 \mathrm{~ns}$. The gear electrode was used to fix a position of a streamer/filament in space; a few hours of training of the electrode system provided star of the filaments all the time from the same points. As a result, it was possible to trace development of filaments changing the delay between the start of the pulse and the ICCD gate. It is seen that for both polarities, the discharge starts as a set of streamers starting at the same time (within $1 \mathrm{ns)} \mathrm{from} \mathrm{the}$ high-voltage electrode. Additional imaging with narrow-band filters show that at the streamer stage, the optical emission from the streamer is mainly emission of the second positive system of molecular nitrogen. Starting from 3-5 ns from the beginning of the pulse, a few protrusions are clearly seen in the images. Starting from 7-9 ns, a set of well-developed filaments is observed on the surface of the dielectric. The emission from the channels is a broad-band CW emission. A question about the optical emission distribution in space during the transition (3-5 ns) remains open.

Figure 4 presents the images taken in the system with the smooth edge electrode by long distance microscope with a long camera gate $(20 \mathrm{~ns})$ at different amplitudes of high voltage pulse. For both polarities, with the increasing amplitude of the voltage, the transition from streamer to filament happens at the specific voltage interval, the values are slightly 
different from the values with gear electrode.Main features of the nanosecond SDBD discharge are illustrated by two left rows, $U= \pm 20 \mathrm{kV}$ and $U= \pm 25 \mathrm{kV}$. Streamers start in the equidistant manner from the electrode; the positive polarity streamers move faster than the negative polarity streamers. During the transition regime, a few channels move faster than the surrounding streamers; the distribution of these channels around the edge of the electrode is arbitrary. At higher voltages, a regular set of filaments is developed. Difference in morphology is linked to the different polarities of the pulse.

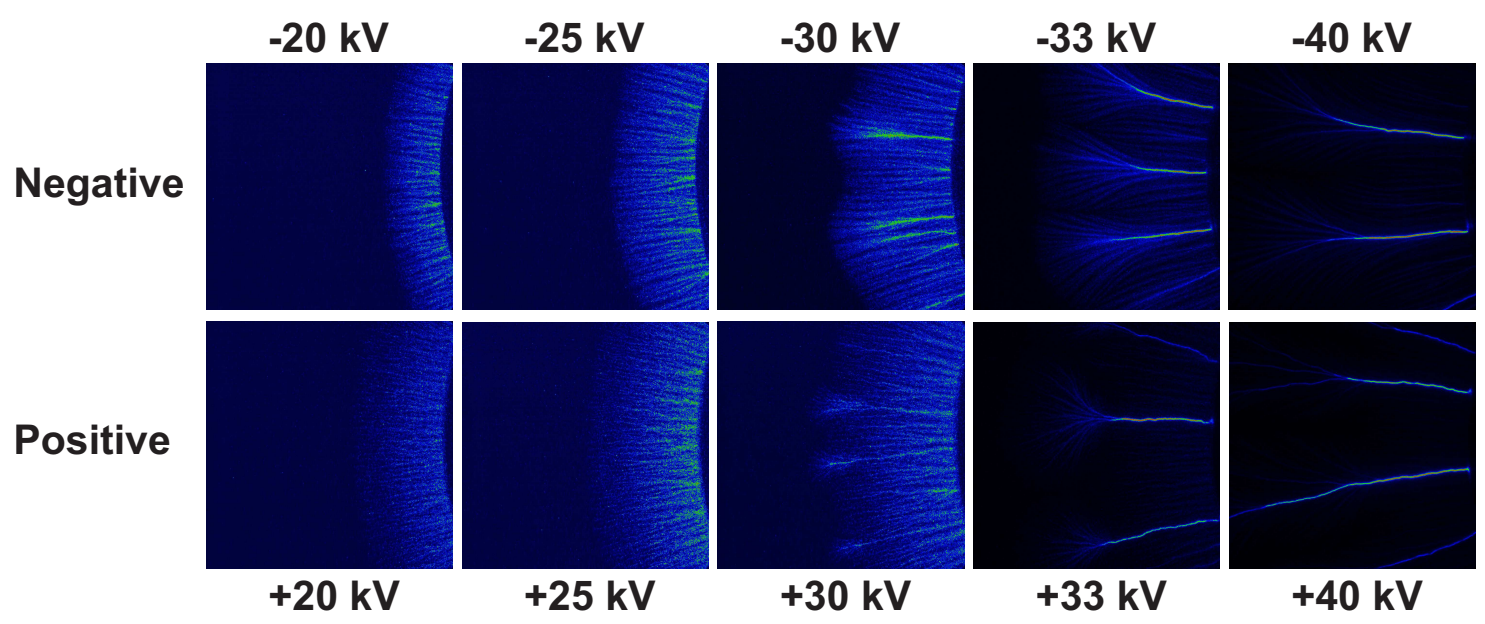

Fig. 4 ICCD images of filamentary discharge for different voltage amplitudes in nitrogen $(P=6 \mathrm{bar})$. Camera gate is $20 \mathrm{~ns}$.

\section{B. Streamer-to-filament transition on different dielectric materials}

Streamer-to-filament transition is as a general feature of the high pressure nanosecond dielectric barrier discharge in molecular gases. The experiments presented here were done for both PVC and ceramic dielectric. Streamer-to-filament transition curves demonstrate the same behavior in both cases.

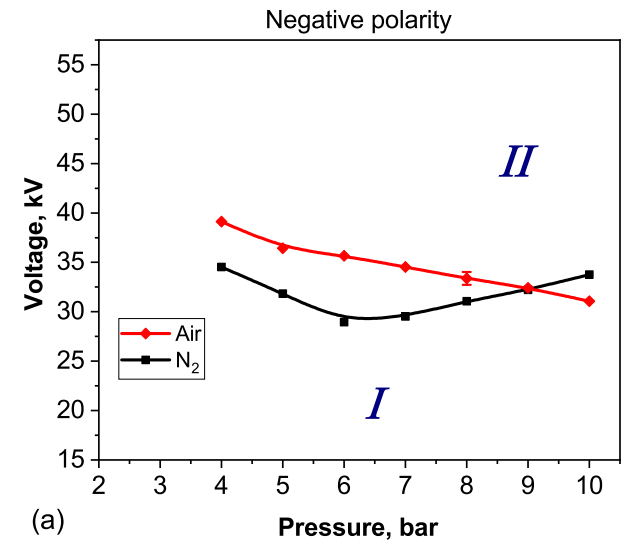

(a)

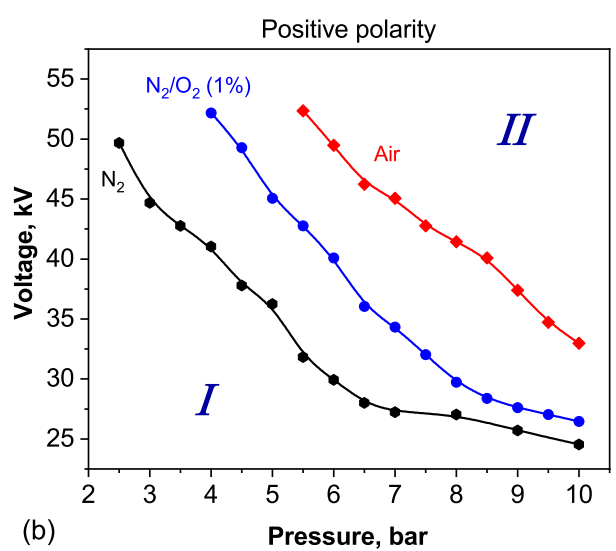

(b)

Fig. 5 Streamer-to-filament transition curves on PVC taken at (a) air and nitrogen for negative polarity and (b) air, nitrogen and the mixture of nitrogen and $1 \%$ of oxygen for negative polarity during first ns of the discharge.

For negative polarity, changing of pressure or the gas mixture composition do not influence significantly the transition voltage. The difference between air and pure nitrogen on PVC dielectric (see Figure 5 ) does not exceed $5 \mathrm{kV}$ which is negligible when comparing to the difference in positive polarity. For positive polarity, the dependence of 
transition voltage upon pressure is sharp, decaying from 50 to $25 \mathrm{kV}$ between 3 and $10 \mathrm{kV}$. Similar behaviour of the transition curves is observed for ceramic as a dielectric (see Figure 6). For all transition curves, we considered a state as a "transition" if 3-5 filaments were identified at the ICCD image for teh ICCD gate 12 ns. This is illustrated by the ICCD images at the right hand side of Figure 6.
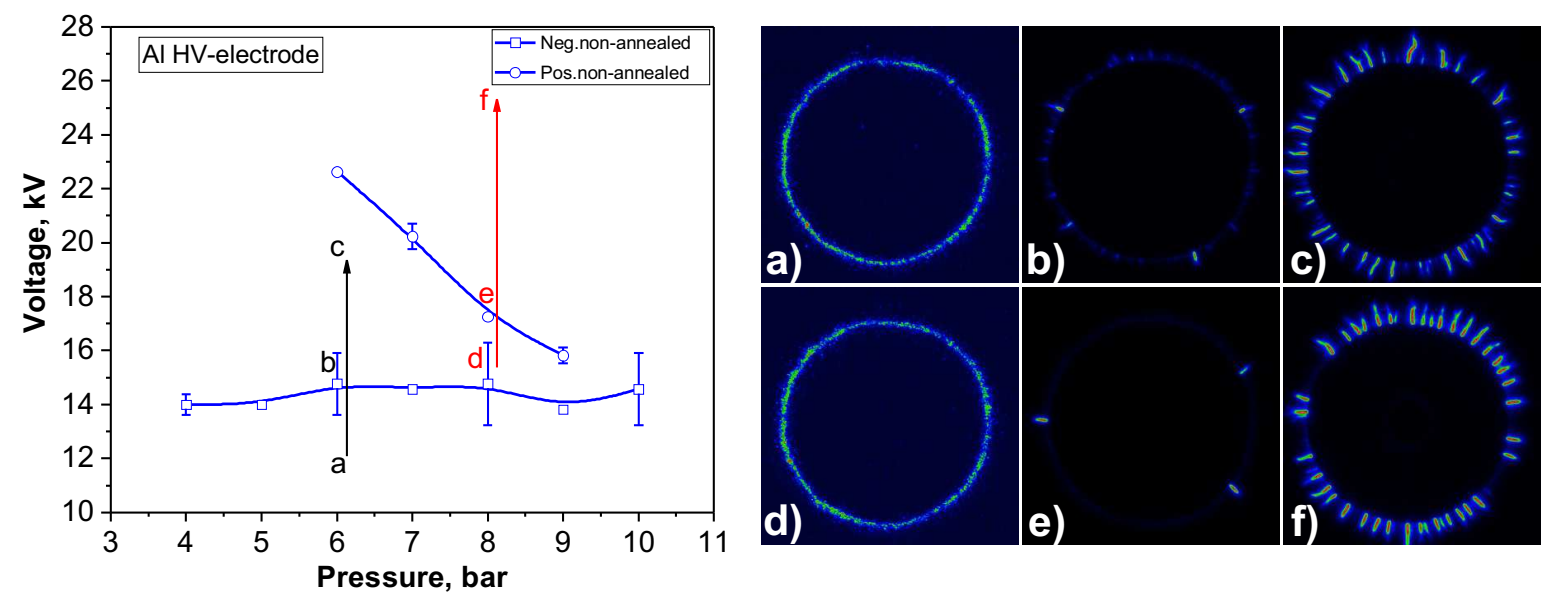

Fig. 6 Streamer-to-filament transition curves on a non-annealed aluminium ceramic electrode and typical ICCD images at the moment of streamer, transition and filament. Camera gate is 12 ns.

The additions of molecular oxygen play an important role in the transition (see Figure 5 ). Addition of $1 \%$ of $\mathrm{O}_{2}$ increases a transition voltage by $10 \mathrm{kV}$, and the difference of the transition voltage between air and pure nitrogen at 6 bar is about $20 \mathrm{KV}$. A possible explanation is that the main process responsible for the appearance of the filaments at the negative polarity is field emission from the edge of the high-voltage electrode with a negligible role of a main gas composition, while at positive polarity the photoionization increases stability of the filaments, providing more distributed in space (non-contracted) discharge at higher voltages.

\section{Deposited energy in the filaments}

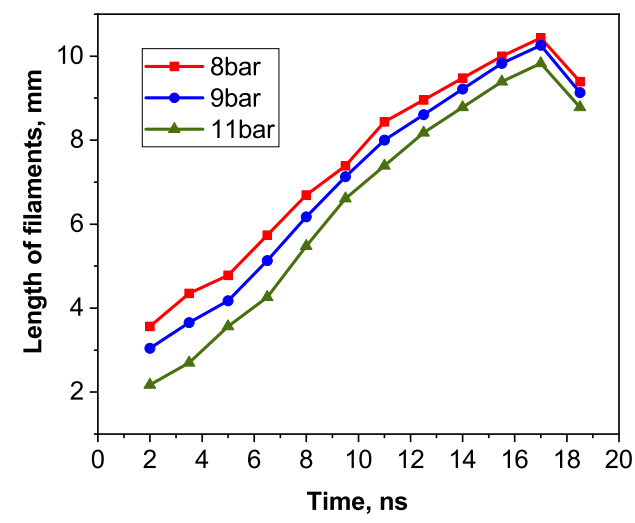

(a)

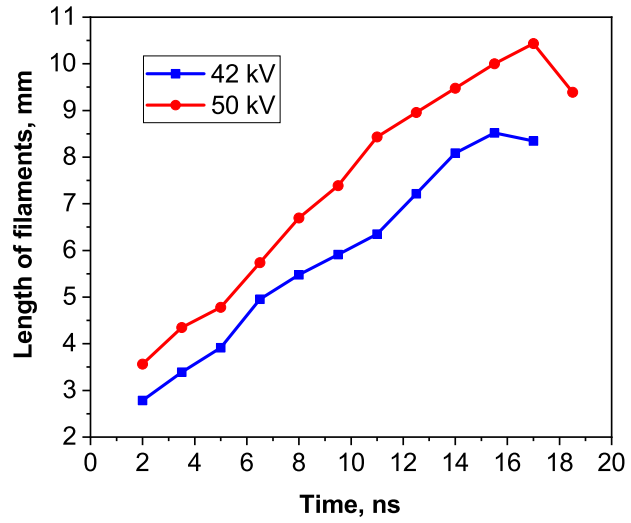

(b)

Fig. 7 x-t diagrams of propagation of filaments for discharge in air at positive polarity: (a) for different pressures; the applied voltage amplitude is $U=+50 \mathrm{kV}$; (b) for different voltages; the pressure is $P=8$ bar. 
To analyze the specific deposited energy, namely the deposited energy per molecule, it is necessary to estimate the total volume of the filaments. So-called $x-t$ diagrams were used to quantify the propagation of the filaments. It can be found that the velocity of the filaments at constant voltage practically does not depend upon pressure (see figure 7 (a)). Different values of the first $x$ points for $P=8,9$ and 11 bar demonstrate that before the transition, streamers cover a longer distance in the case of lower pressure. Being moved at the same initial point, the $x-t$ diagrams for different pressures will follow almost the same way; the velocity of the filament propagation before slowing down is about $0.4 \mathrm{~mm} / \mathrm{ns}$. On the trailing edge of the voltage pulse, at $t>15 \mathrm{~ns}$, the velocity of the filaments decreases. To some extent, it is possible to increase the length of the filaments by increasing the duration of the pulse.

When the pressure is fixed and the voltage amplitude is changed (see figure 7(b)), the velocity of filaments is not constant any more: increase of the voltage amplitude from $U=+42 \mathrm{kV}$ to $U=+50 \mathrm{kV}$ leads to acceleration of the filaments from $0.3 \mathrm{~mm} / \mathrm{ns}$ to $0.5 \mathrm{~mm} / \mathrm{ns}$. The regime with pronounced difference in the velocity of filaments, constant pressure $P=8$ bar and the voltage amplitude changing between $U=+42 \mathrm{kV}$ and $U=+50 \mathrm{kV}$ was selected to study the specific deposited energy.

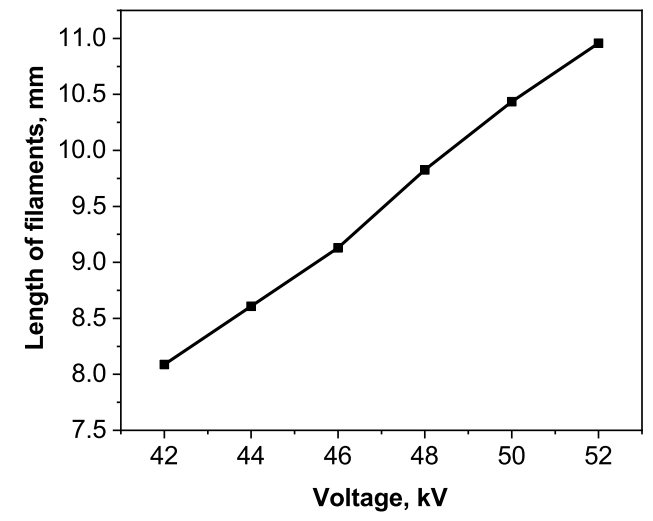

(a)

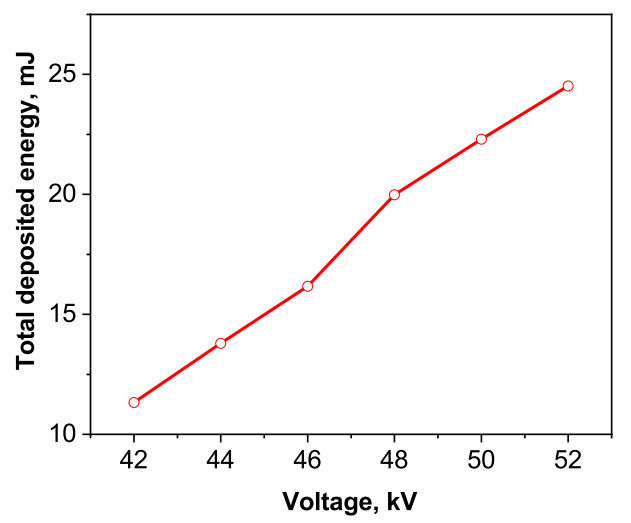

(b)

Fig. 8 A maximum length of the filaments propagation (a) and the total deposited energy (b) as a function of the voltage amplitude. Air, positive polarity of the pulse, $P=8$ bar.

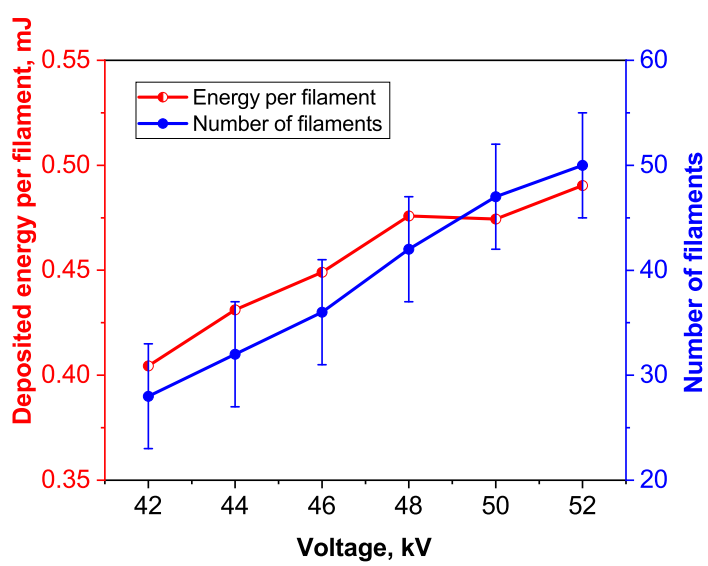

(a)

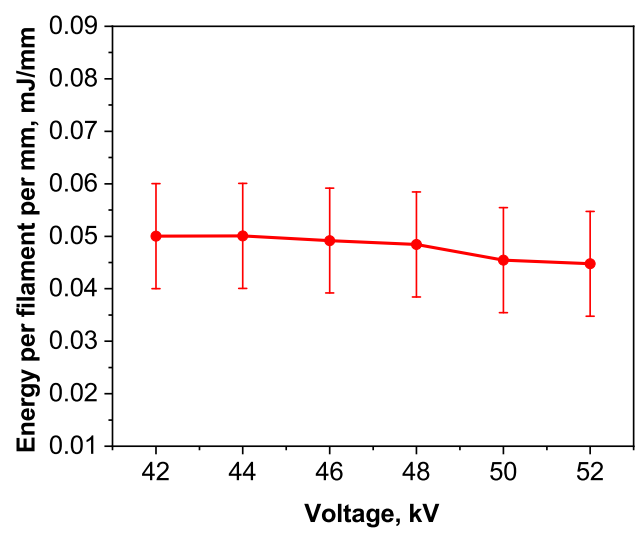

(b)

Fig. 9 Number of filaments and the deposited energy per filament (a); the energy per filament per mm (b) as a function of the voltage amplitude. Air, positive polarity of the pulse, $P=8$ bar.

A maximum length of the filaments propagation at different voltage amplitudes is presented in figure 8 (a). The 
length increases from $8.1 \mathrm{~mm}$ at $U=+42 \mathrm{kV}$ to $11 \mathrm{~mm}$ at $U=+52 \mathrm{kV}$. The total deposited energy measured from the waveforms obtained by a back current shunt, also increases with voltage, from $11.2 \mathrm{~mJ}$ at $U=+42 \mathrm{kV}$ to $24.6 \mathrm{~mJ}$ at $U=+52 \mathrm{kV}$ (figure 8 (b)). The last parameter to be taken into account is the number of filaments increasing from 30 at $U=+42 \mathrm{kV}$ to 50 at $U=+52 \mathrm{kV}$ (figure 9 (a)). Assuming that the energy is equally distributed between the filaments, will obtain that the energy changes from 0.40 to $0.48 \mathrm{~mJ}$ per filament with voltage. Finally, assuming that the energy is distributed uniformly along the filament, and dividing the energy per filament by the filament length, will get the plot presented in figure 9 (b). It is clearly seen that in spite of strong dependence of parameters on voltage, the energy per unit length is almost constant for all voltage amplitudes under study.

The cross section data of the filament $(18 \pm 2 \mu \mathrm{m}$ in diameter) estimated in previous work [9] can be used to determine the specific delivered energy. In assumption that almost all the delivered energy is spent in the core of the filament and almost no energy is spent in the streamer shell, one can estimate the energy per molecule at taking the number of molecules being equal to the number of molecules at $P=8$ bar and $T=300 \mathrm{~K}$. For the energy per unit length $\sim 0.05 \mathrm{~mJ} / \mathrm{mm}$ as obtained for the conditions of the present work figure 9 (b), will obtain the specific delivered energy $\omega \approx 6.4 \mathrm{eV} /$ particle.

\section{Particle Image Velocimetry (PIV) measurement}

The PIV measurement can formally be performed from the initial stage of the flow evolution - the formation of the cylindrical shocks driven by the filament. However, the detailed analysis of the data obtained during this stage show a significant influence of the aero-optical distortions driven by the shock-associated density gradients.

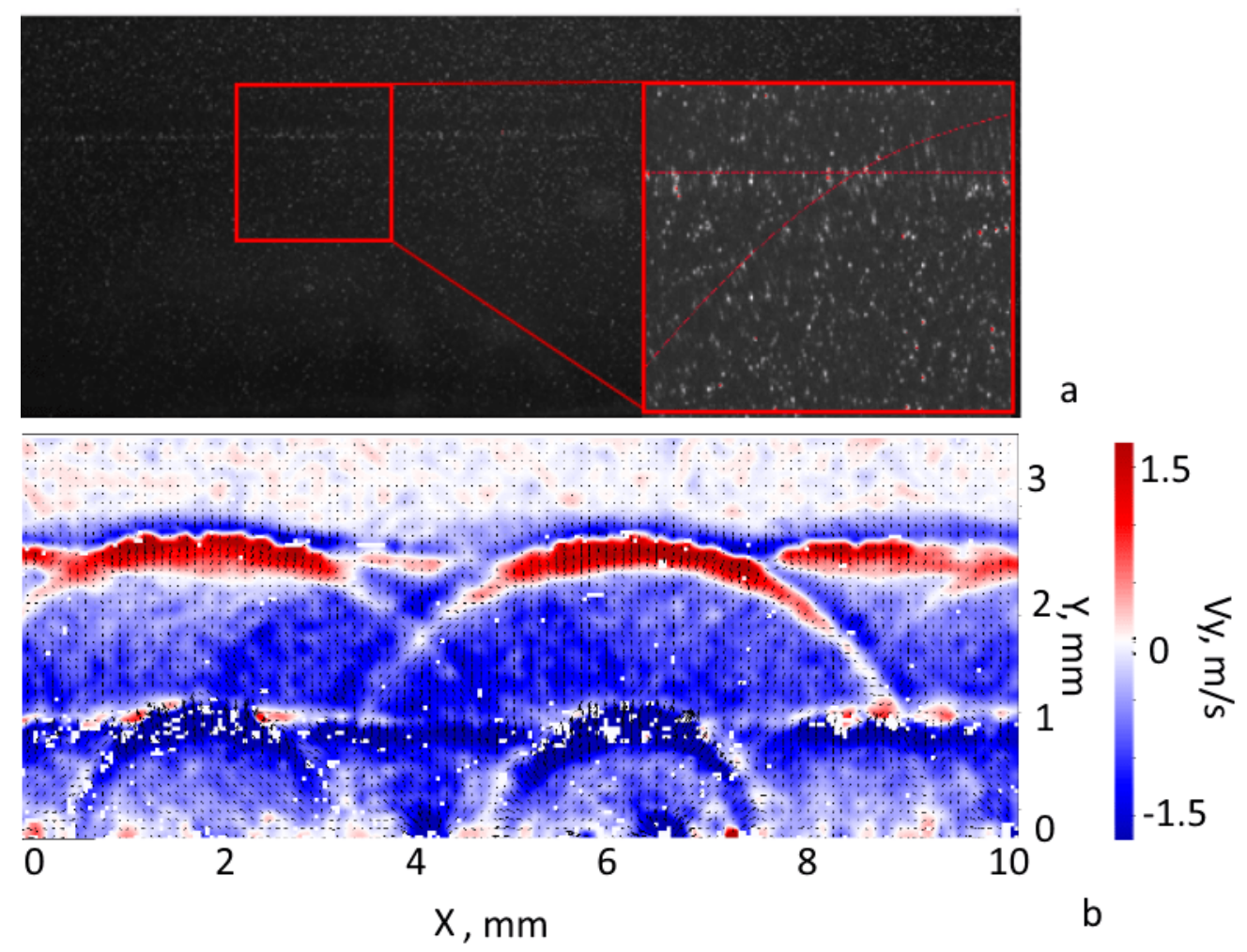

Fig. 10 PIV analysis: (a) particle images near the shock front; (b) PIV image of cylindrical shock waves

Typical velocity field obtained during the stage of shocks propagation is shown in Figure $10 \mathrm{p}$. It can be seen that the cylindrical shock waves are formed by the individual filaments. These cylindrical shocks are superimposed on the plain wave, generated by the initial streamer discharge.

The detailed analysis of PIV data at this stage shows the unphysical structure of the velocity field near the shock front. Furthermore, the increase of the delay between the two laser pulses leads to the splitting of the shock into two separate waves, indicating that the obtained vector field does not correspond to displacement of the seed particles. The 
close analysis of the particle images near the shock front shows a significant smearing of the particles (Figure 10a). The smearing is a result of the refraction of the light in the density gradient near the shock. Smearing of the particles is interpreted by the PIV algorithm as physical displacement of the particles. The aero-optical distortions are known in PIV measurements [10] and sometimes can used for the quantitative density field characterization. In our case the refraction leads to the aero-optical error on the order of $1 \mathrm{px}$, that corresponds to the absolute velocity error of $2 \mathrm{~m} / \mathrm{s}$.

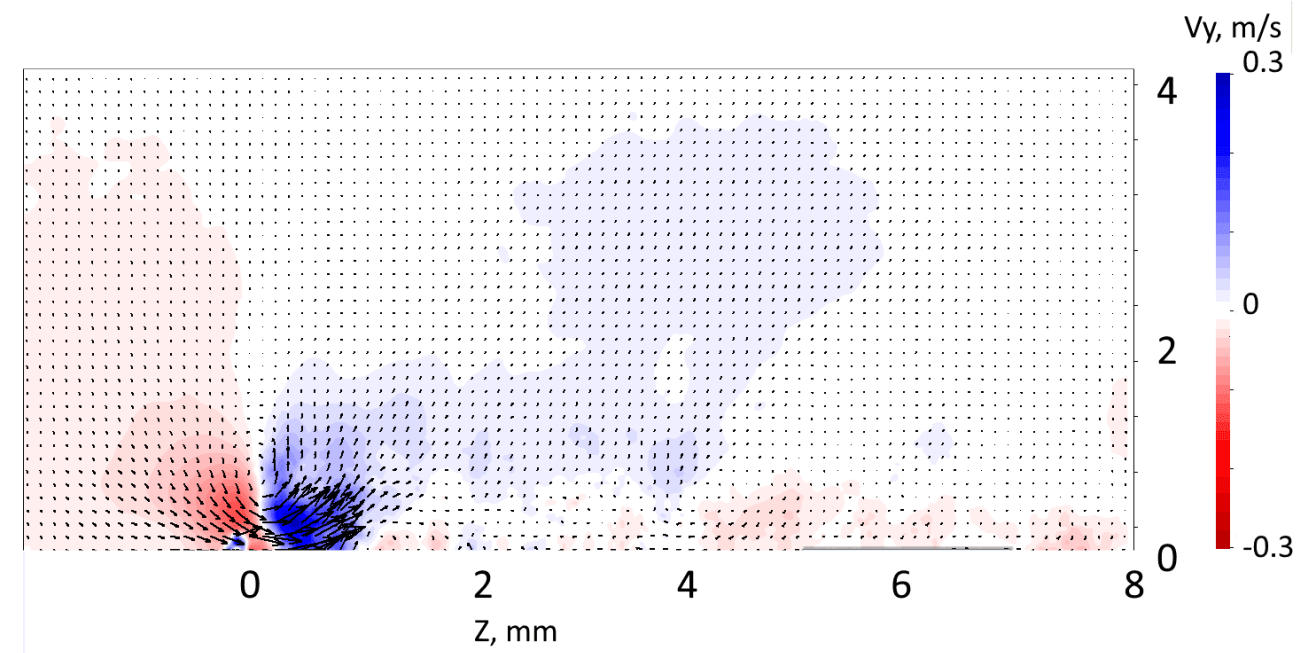

Fig. 11 PIV image of the flow structure induced after the streamer discharge.

The structure of the flow after the shock propagation stage was studied in a similar optical configuration. Long after the pulse, the density gradients are much lower than during the shock propagation stage, thus the aero-optical error is assumed to be unsignificant at this stage. The flow structure induced after the streamer discharge is shown in Figure 11 . One can see that a single vortex is formed above the exposed electrode at a typical time $100 \mu$ s. The velocity magnitude in the vortex is $0.2-0.5 \mathrm{~m} / \mathrm{s}$. In a circular electrode configuration, this corresponds to the formation of a toroidal vortex.

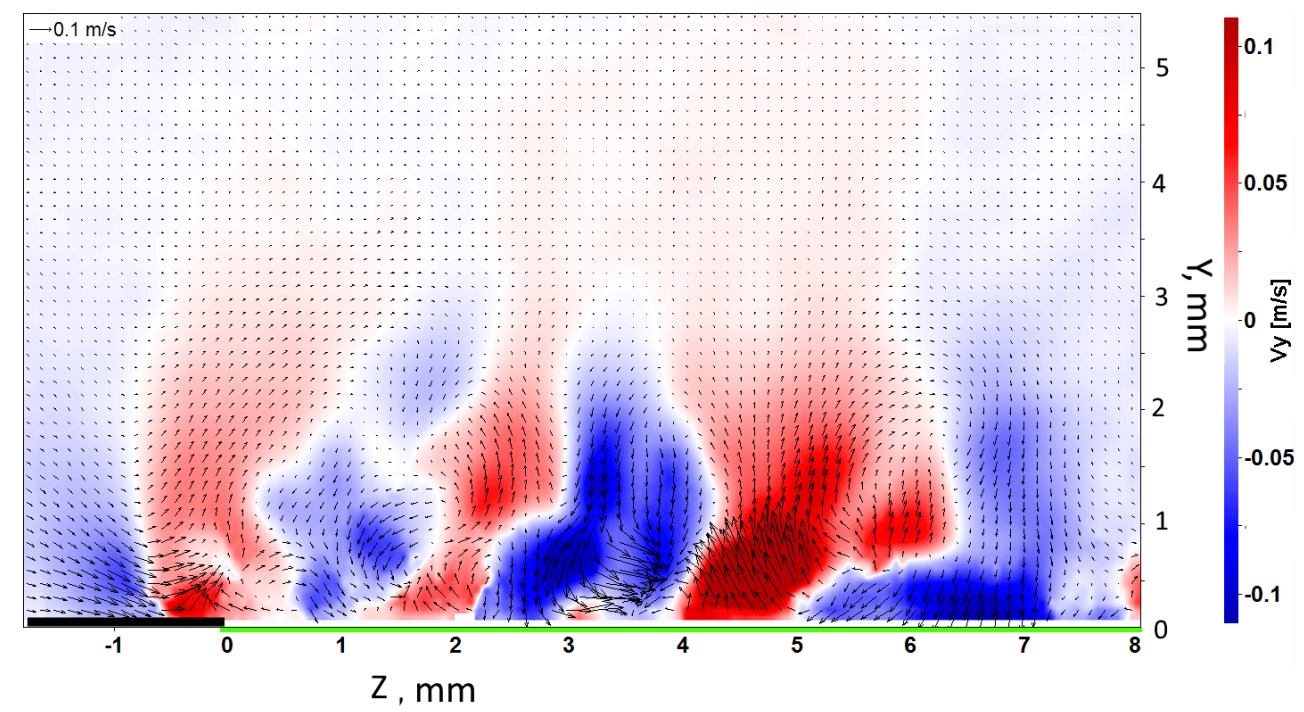

Fig. 12 PIV image of the flow structure induced after the filamentary discharge.

To analyze the flow induced by a constricted discharge, the electrode system was modified. The ground electrode was manufactured of a single, $2 \mathrm{~mm}$ wide strip of the metal foil to stabilize a position of the discharge channel (although 
some fluctuations in the filament geometry were still present). Constriction of the discharge leads to the formation of the three-dimentional flow structures with a typical scale of 1-2 mm (Figure 12). The perturbation are present along the whole length of the filament. A typical velocity magnitude in the given cross-section varies from pulse to pulse, but never exceeds the value of $0.5 \mathrm{~m} / \mathrm{s}$.
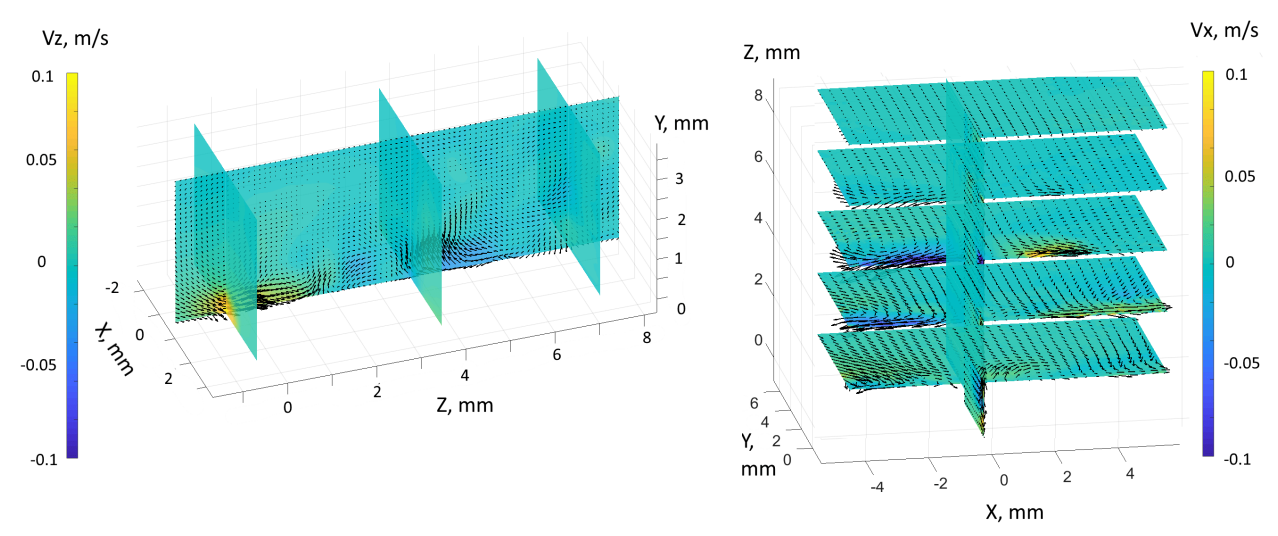

Fig. 13 A global structure of the flow reconstructed from the averaged velocity.

A global structure of the flow was reconstructed from the averaged velocity fields and is shown in Figure 13 The efforts to stabilize the position of the filament provided the accuracy of the channel position within 1-2 mm for the long distances from the electrode and about $0.5 \mathrm{~mm}$ at the electrode. Therefore, the averaged data presents rather a typical flow structure; due to the variations of the filaments pattern, a typical velocity magnitude in the close vicinity of the channel is underestimated. Key features obtained during the cavern decay are the jets propagating along the filaments starting from first 100-200 $\mu \mathrm{s}$. These jets are assumed to be generated due to the baroclinic torque present near the end of the heat release zone. Similar structures were obtained during the cavern decay of the volumetric electrode and laser sparks [11, 12]. Another typical feature is the jet propagating along the surface in the crossflow planes.

\section{Conclusion}

Experimental study of streamer-to-filament transition in molecular nitrogen and nitrogen:oxygen mixtures for the electrode systems with PVC or ceramics as dielectric has been presented. Two polarities of the discharge were compared via microimaging and analysis of streamer-to-filament transition curves for voltage and pressure. It was shown experimentally that, for positive polarity, the velocity of filaments changes with the applied voltage but almost does not change with pressure. For pressure and voltage ranges indicated above, the velocity of filaments stays between $0.3 \mathrm{~mm} / \mathrm{ns}$ and $0.5 \mathrm{~mm} / \mathrm{ns}$. The specific delivered energy in the filamentary plasma measured from experiment can be as high as $\omega=6.4 \mathrm{eV} /$ particle. The flow structure induced by a nanosecond SDBD was studied using particle image velocimetry. In the streamer mode the discharge creates a single vortex, formed in the vicinity of the exposed electrode. In the filamentary mode complex three-dimentional turbulence with a typical scale of 1-2 $\mathrm{mm}$ is present near the surface during 0.2-2 ms after the discharge pulse. The average flow induced by a single constricted filament have the features typical for the decay of the low density caverns of the electrode and laser sparks: formation of the jets along the filament near the ends of the filament and in the spanwise direction near its central part. A typical velocity magnitude for the flow induced by the filamentary nSDBD was within $0.5 \mathrm{~m} / \mathrm{s}$.

\section{Acknowledgments}

The work was partially supported by LabEx Plas@ Par, convention Ecole Polytechnique-DGA 2790, French National Research Agency (ASPEN Project), the French-Russian international laboratory LIA KaPPA "Kinetics and physics of pulsed plasmas and their afterglow" (RFBR project 17-58-16004 and CNRS financial and organization support). The authors are thankful to Ali Mahjoub, Pascal Pariset and Bruno Dufour for engineering support. 


\section{References}

[1] Starikovskaia, S., Allegraud, K., Guaitella, O., Kosarev, I., Mintusov, E., Pendleton, S. J., Popov, N., Sagulenko, P., and Rousseau, A., "Surface discharges: possible applications for plasma-assisted ignition and electric field measurements," 48th AIAA Aerospace Sciences Meeting Including the New Horizons Forum and Aerospace Exposition, 2010, p. 1587.

[2] Kosarev, I., Khorunzhenko, V., Mintoussov, E., Sagulenko, P., Popov, N., and Starikovskaia, S., "A nanosecond surface dielectric barrier discharge at elevated pressures: time-resolved electric field and efficiency of initiation of combustion," Plasma Sources Science and Technology, Vol. 21, No. 4, 2012, p. 045012.

[3] Stepanyan, S., Starikovskiy, A. Y., Popov, N., and Starikovskaia, S., "A nanosecond surface dielectric barrier discharge in air at high pressures and different polarities of applied pulses: transition to filamentary mode," Plasma Sources Science and Technology, Vol. 23, No. 4, 2014, p. 045003.

[4] Shcherbanev, S., Khomenko, A. Y., Stepanyan, S., Popov, N., and Starikovskaia, S., "Optical emission spectrum of filamentary nanosecond surface dielectric barrier discharge," Plasma Sources Science and Technology, Vol. 26, No. 2, 2017 , p. 02 LT01.

[5] Shcherbanev, S. A., Popov, N. A., and Starikovskaia, S. M., "Ignition of high pressure lean $\mathrm{H}_{2}$ : air mixtures along the multiple channels of nanosecond surface discharge," Combustion and Flame, Vol. 176, 2017, pp. 272-284.

[6] Van der Horst, R., Verreycken, T., Van Veldhuizen, E., and Bruggeman, P., "Time-resolved optical emission spectroscopy of nanosecond pulsed discharges in atmospheric-pressure $\mathrm{N}_{2}$ and $\mathrm{N}_{2} / \mathrm{H}_{2} \mathrm{O}$ mixtures," Journal of Physics D: Applied Physics, Vol. 45, No. 34, 2012, p. 345201.

[7] Lo, A., Cessou, A., Lacour, C., Lecordier, B., Boubert, P., Xu, D., Laux, C., and Vervisch, P., "Streamer-to-spark transition initiated by a nanosecond overvoltage pulsed discharge in air," Plasma Sources Science and Technology, Vol. 26, No. 4, 2017, p. 045012 .

[8] Orrière, T., Moreau, E., and Pai, D. Z., "Ionization and recombination in nanosecond repetitively pulsed microplasmas in air at atmospheric pressure," Journal of Physics D: Applied Physics, Vol. 51, No. 49, 2018, p. 494002.

[9] Shcherbanev, S., Ding, C., Starikovskaia, S., and Popov, N., "Filamentary nanosecond surface dielectric barrier discharge. Plasma properties in the filaments," Plasma Sources Science and Technology, 2019. Accepted.

[10] Elsinga, G. E., van Oudheusden, B. W., and Scarano, F., "Evaluation of aero-optical distortion effects in PIV," Experiments in fluids, Vol. 39, No. 2, 2005, pp. 246-256.

[11] Singh, B., Rajendran, L. K., Giarra, M., Vlachos, P. P., and Bane, S. P., "Measurement of the flow field induced by a spark plasma using particle image velocimetry," Experiments in Fluids, Vol. 59, No. 12, 2018, p. 179.

[12] Firsov, A. A., Isaenkov, Y. I., Shurupov, M. A., and Leonov, S. B., "Development of plasma actuator based on surface sparks for a buffet control," 54th AIAA Aerospace Sciences Meeting, 2016, p. 1690. 